OPEN ACCESS

Edited by:

Yong Zhao,

Institute of Zoology (CAS), China

Reviewed by:

Lianjun Zhang,

Suzhou Institute of Systems Medicine,

China

Irun R. Cohen,

Weizmann Institute of Science, Israe

*Correspondence:

Huanfa Y

yihuanfa@jlu.edu.cn

tThese authors have contributed equally to this work

Specialty section This article was submitted to Immunological Tolerance and Regulation,

a section of the journal Frontiers in Immunology

Received: 18 May 2018 Accepted: 04 October 2018 Published: 09 November 2018

Citation:

Wang X, Qiu L, Li Z, Wang X-Y and $Y i H$ (2018) Understanding the Multifaceted Role of Neutrophils in Cancer and Autoimmune Diseases.

Front. Immunol. 9:2456.

doi: 10.3389/fimmu.2018.02456

\section{Understanding the Multifaceted Role of Neutrophils in Cancer and Autoimmune Diseases}

\author{
Xu Wang ${ }^{1,2,3,4+}$, Lin Qiu ${ }^{1,2,3,4+}$, Ziyi Li ${ }^{2,3}$, Xiang-Yang Wang ${ }^{5,6}$ and Huanfa Yi ${ }^{1,2,3 *}$ \\ ${ }^{1}$ Central laboratory of Eastern Division, The First Hospital of Jilin University, Changchun, China, ${ }^{2}$ Institute of Immunology, Jilin \\ University, Changchun, China, ${ }^{3}$ National-local Joint Engineering Laboratory of Animal Models for Human Diseases, \\ Changchun, China, ${ }^{4}$ Department of Neurology, The First Hospital of Jilin University, Changchun, China, ${ }^{5}$ Department of \\ Human and Molecular Genetics, Virginia Commonwealth University, Richmond, VA, United States, ${ }^{6}$ Massey Cancer Center, \\ Virginia Commonwealth University, Richmond, VA, United States
}

Neutrophils are one of the first immune cell types that are recruited to injury and infection site. As a vital component of the immune system, neutrophils are heterogeneous immune cells known to have phagocytic property and function in inflammation. Recent studies revealed that neutrophils play dual roles in tumor initiation, development, and progression. The multifunctional roles of neutrophils in diseases are mainly due to their production of different effector molecules under different conditions. N1 and N2 neutrophils or high density neutrophils (HDNs) and low density neutrophils (LDNs) have been used to distinguish neutrophils subpopulations with pro- vs. anti-tumor activity, respectively. Indeed, N1 and N2 neutrophils also represent immunostimulating and immunosuppressive subsets, respectively, in cancer. The emerging studies support their multifaceted roles in autoimmune diseases. Although such subsets are rarely identified in autoimmune diseases, some unique subsets of neutrophils, including low density granulocytes (LDGs) and $\mathrm{CD} 177^{+}$neutrophils, have been reported. Given the heterogeneity and functional plasticity of neutrophils, it is necessary to understand the phenotypical and functional features of neutrophils in disease status. In this article, we review the multifaceted activates of neutrophils in cancer and autoimmune diseases, which may support new classification of neutrophils to help understand their important functions in immune homeostasis and pathologies.

Keywords: neutrophils, cancer, N1/N2, autoimmune diseases, immune tolerance

\section{SUMMARY POINTS}

- Neutrophils play multifaceted functions under different pathological conditions by releasing various effector molecules and cytokines. Neutrophils are more heterogeneous than previously thought and different subpopulations have distinct activities in diseases.

- Neutrophils in cancer could be divided into N1 and N2 subsets, or high density neutrophils, and low density neutrophils based on functional characteristics.

- Neutrophils in certain autoimmune diseases may also be classified into different subsets, with low density granulocytes (LDGs) representing pro-inflammatory neutrophils and $\mathrm{Gr}-1^{\text {high }}$ or CD177 neutrophils exhibiting anti-inflammatory effects. 


\section{INTRODUCTION}

Neutrophils are the most abundant leukocytes in the circulating system, making up $50-70 \%$ of the whole white blood cells in human $(1,2)$. They constitute the first line of defense and protect the host from pathogen assaults via multiple mechanisms including phagocytosis, release of granules, production of cytokines, and formation of neutrophil extracellular traps (NETs) (3). Neutrophils are not only an important component of innate immunity, but also participate in regulation of adaptive immunity through interplays with various adaptive immune cells. In addition to the host defense, neutrophils are also involved in the pathogenesis of many diseases, including cancer and autoimmune disorders (2).

Although neutrophils have long been found to be present in different type of tumors, these tumor-associated neutrophils (TANs) were believed to be functionally neutral due to their short lifespan (2). Increasing studies over the past few years began to reveal the differential roles of neutrophils in cancer. Only recently, the level of neutrophils in the tumor tissues was considered as a marker for a poor prognosis of cancer patients (4). According to their functions, TANs are divided into two subgroups with anti-tumor (N1) or pro-tumor (N2) activity (5$7)$, which is similar to tumor-associated macrophages $(8,9)$. Recently, it was suggested that circulating neutrophils can also be classified into high density neutrophils (HDNs) and low density neutrophils (LDNs), which functionally mirrors N1 and N2 neutrophils, respectively $(10,11)$.

While neutrophils have also been reported to show multifaceted functions in many autoimmune diseases (12-17), few researchers have attempted to distinguish subpopulations of neutrophils in autoimmune diseases. Most immune cells, including $\mathrm{T}$ cells, dendritic cells, and macrophages have been classified into different subsets that exert different or even opposite roles in different disease contexts (18-21). In this article, we summarize our current understanding of neutrophils, the most abundant leukocytes in circulation, and their multifaceted functions in different diseases, particularly autoimmune conditions. We describe the various effector molecules produced by neutrophils that define its functions in a disease-specific context, which may provide some insights into the potential classification of neutrophils.

\section{THE DIVERSITY OF NEUTROPHILS IN CANCERS}

Cancer cells can produce various chemokines and cytokines, which recruit neutrophils to the tumor milieu $(22,23)$. Although neutrophils have previously thought to be terminally differentiated cells due to their short life span $(24,25)$, the plasticity of neutrophils has been unveiled in the recent years. These cells may be divided into different subsets based on their differential effects on cancer initiation, development, and progression. Neutrophils in cancer, also known as tumor associated neutrophils (TANs), are functionally classified as tumor-suppressing $\mathrm{N} 1$ or tumor-promoting $\mathrm{N} 2$ phenotype.
Alternatively, they can be divided into high density neutrophils (HDNs) and low density neutrophils (LDNs) based on the density of circulating neutrophils in cancer patients.

$\mathrm{N} 1$ and $\mathrm{N} 2$ subpopulations of TANs display distinct functions in cancer. N1 neutrophils have potent anti-tumor activity mainly due to their release of pro-inflammatory or immunostimulatory cytokines, such as interleukin (IL)-12, tumor necrosis factor (TNF)- $\alpha$, CCL3, CXCL9, CXCL10, which facilitates recruitment and activation of $\mathrm{CD}^{+} \mathrm{T}$ cells $(26,27)$. In contrast, N2 neutrophils have strong immunosuppressive and tumorpromoting activity, including promotion of tumor angiogenesis, invasion and metastases via various factors, such as hepatocyte growth factor (HGF) (28), oncostatin M (6), reactive oxygen species (ROS) (29), reactive nitrogen species (RNS) (29), matrix metalloproteinase (MMPs) (30), and neutrophils elastase (NE) $(4,30)$. TANs were shown to locate at the margin of tumor site in early stage cancer, but they can massively infiltrate into the center of tumor at late stage (31). Research in mouse lung carcinoma and mesothelioma models suggests that TANs have a tumorsuppressing N1 phenotype at the early stage of tumor, whereas they convert into a tumor-promoting N2 phenotype during tumor progression (31). Such a phenotypic transformation may be induced by the factors produced by cancer cells and/or other immune cells in the tumor microenvironment. Recent studies have shown that the cytokine TGF- $\beta$ and type I interferons are major factors involved in polarization of neutrophils. In the presence of TGF- $\beta$, neutrophils are skewed toward an N2 phenotype, whereas the blockade of TGF- $\beta$ facilitates neutrophil development into an $\mathrm{N} 1$ phenotype (32). In contrast, type I interferons polarize neutrophils to an N1 phenotype while the impaired type I interferon signal results in polarization of neutrophils to an N2 phenotype (33). A recent study reported that angiotensin converting enzyme inhibitors (ACEI) or angiotensin II type I receptor (AGTR1) antagonist also promoted an N1 phenotype of neutrophils, which was association with inhibition of tumor growth. However, addition of angiotensin II reversed this process (34). Furthermore, ACEI treatment resulted in a reduction of serum TGF- $\beta$ in tumor-bearing mice, suggesting that angiotensin II regulates neutrophils polarization through induction of TGF- $\beta$ and further underscores an important role of TGF- $\beta$ in N1-N2 polarization.

HDNs and LDNs represent another classification for circulating neutrophils in cancer paients. Density gradient centrifugation is the most common and validated approach to separate mononuclear and polymorphonuclear leukocytes (10). Mature neutrophils were thought to exist in the high density sedimentary fraction of leukocytes with segmented nucleus (35). However, recent findings showed that some neutrophils also exist in the low density fraction $(11,36)$. In addition to classification of HDNs and LDNs in blood, LDNs can further be divided into mature and immature populations based on the shape of their nucleus. The latter is also known as granulocytic-myeloid derived suppressor cells (G-MDSCs), which have a characteristic of banded or ring-shaped nucleus (37-39). Compared with HDNs, LDNs show a larger cellular size, reduced anti-tumor cytotoxicity, decreased phagocytic activity, impaired migratory capacity, and less oxidative burst, instead, these cells exhibit 
highly anti-inflammatory and tumor-promoting activity (11). In this context, HDNs are functionally similar to N1 neutrophils, while LDNs resemble N2 neutrophils. Interestingly, HDNs can be converted into LDNs by TGF- $\beta$ (11), indicating the plasticity of these cells. However, this phenomenon was only observed in HDNs from tumor-bearing mice not those from tumor-free mice (11), suggesting that HDNs may need to be primed before their conversion into LDNs in the presence of TGF- $\beta$. Given the functional similarity of LDNs and N2 neutrophils, they are likely to belong to the same subpopulations of neutrophils that are present in different sites. However, it is difficult to test this possibility due to a lack of validated surface markers currently available to define N2 neutrophils or LDNs. Therefore, further investigation is necessary to understand the developmental relationship between N1/N2 neutrophils and HDNs/LDNs and, more importantly, to phenotypically identify these different subtypes of neutrophils as well as signals or factors involved in regulation of their polarization.

\section{THE DUAL ROLES OF NEUTROPHILS IN CANCERS}

As an important immune cell type, neutrophils are present in circulation and tumor milieu. These cells display either pro- or anti-tumor activity in a context-specific manner (Figure 1).

The anti-tumor activity of neutrophils is executed mainly through antibody-dependent cellular cytotoxicity (ADCC) and target-specific antibody cytotoxicity $(40,41)$. Many potential cytotoxic mediators produced by neutrophils, including TNF$\alpha$, NO, and $\mathrm{H}_{2} \mathrm{O}_{2}$, can contribute directly to their tumorsuppressive activity $(6,31)$. Certainly cytokines, such as GMCSF and IFN- $\gamma$, upregulate co-stimulatory molecules (e.g., CD86, CD54, OX40L, and 4-1BBL) on neutrophils at the early stage of tumorigenesis, which enable these cells to function as antigenpresentation cells for priming proliferation and activation of tumor-reactive T cells $(42,43)$.

The role of neutrophils have been described in various cancer types, including colorectal cancer $(44,45)$, glioblastoma (46), hepatocellular carcinoma (47), renal cell carcinoma (48), melanoma $(49,50)$, pancreatic ductal carcinoma (51), and head and neck cancer (52). Some studies have shown that increased number of neutrophils in tumor tissue or elevated ratio of neutrophil vs. lymphocyte in peripheral blood is associated with a poor prognosis of cancer patients $(45-49,51,52)$. More specifically, neutrophils exhibit their tumor-promoting effect by facilitating tumor initiation, invasion, angiogenesis, and metastasis. Firstly, neutrophils can promote tumor initiation through production of reactive oxygen species (ROS) and reactive nitrogen species (RNS). These reactive radicals are likely to cause DNA damage and genetic instability in some models of chemical induced carcinogenesis (29). In addition, neutrophils accelerate tumor growth and progression by secreting a variety of mediators (4). Neutrophils produce certain growth factors, such as hepatocyte growth factor (HGF), to enhance invasion of human pulmonary adenocarcinoma cells (28). Interestingly, HGF induces release of $\mathrm{NO}$ by neutrophils in a autocrine fashion to kill cancer cells, suggesting a negative feedback loop involved in interplays between neutrophils and transformed cells during tumor progression (53). Neutrophils can also release oncostatin $M$ and matrix metalloproteinase (MMP-9) to induce production of vascular endothelial growth factor, which can promote angiogenesis and invasion of tumor cells in multiple cancer models (54-56). Furthermore, NE and MMPs released by neutrophils promote tumor progression by remodeling tumor extracellular matrix (ECM) (30). Similar to G-MDSCs, neutrophils can also produce arginase 1 to inhibit effector function of T cells (57). In patients with gastric cancer, neutrophils display $\mathrm{T}$ cell suppressive activity in association with surface expression of the immune checkpoint molecule PD-L1or programmed death-ligand 1, which is activated by GM-CSF via the JAK and STAT3 signaling pathways (54-56).

Neutrophils extracellular traps (NETs) are special structure formed by neutrophils, and consist of chromatin and antimicrobial granule proteins, including $\mathrm{NE}$, cathepsin $\mathrm{G}$, and myeloperoxidase (MPO). The presence of NETs within tumors was reported to correlate with poor outcomes of cancer patients (52). NETs can execute their tumor-promoting activity by facilitating proliferation and inhibiting apoptosis of cancer cells (4). Although the precise mechanism remains unclear, this pro-tumor effect of NETs is believed to involve several tumor-promoting molecules, e.g., NE, cathepsin G and MMP-9 (58). NETs can also enhance adhesion of circulating tumor cells to promote metastasis of lung carcinoma (49). Of note, NETs was also shown to reduce the threshold of $\mathrm{T}$ cell activation through direct contact and/or TCR signaling, thereby promoting an adaptive immune response that may help eliminate cancer cells (59). However, the definite evidence supporting NETs-mediated anti-tumor activity is still lacking.

\section{NEUTROPHILS IN AUTOIMMUNE DISEASES}

Accumulating evidence supports the involvement of neutrophils in pathogenesis of many autoimmune diseases. They can influence autoimmune processes either directly via various effector molecules or indirectly through interactions with other immune cells (Table 1). Indeed, neutrophils are believed to be a major cause for induction of autoantibodies in certain autoimmune diseases (2). It is now recognized that neutrophils display phenotypic or functional abnormalities in diverse autoimmune diseases.

\section{Multiple Sclerosis}

Study of experimental autoimmune encephalomyelitis (EAE), a widely used mouse model of human multiple sclerosis (MS) showed that the number of neutrophils increased significantly at the acute phase but declined at the remission phase in the lesion (82). Administration of anti-Ly6G or anti-Gr-1 antibody to deplete neutrophils limits EAE development, suggesting that neutrophils may play a detrimental role in EAE or MS. In contrast, use of G-CSF to promote recruitment and activation of neutrophils can exacerbate EAE (83). A recent study showed 


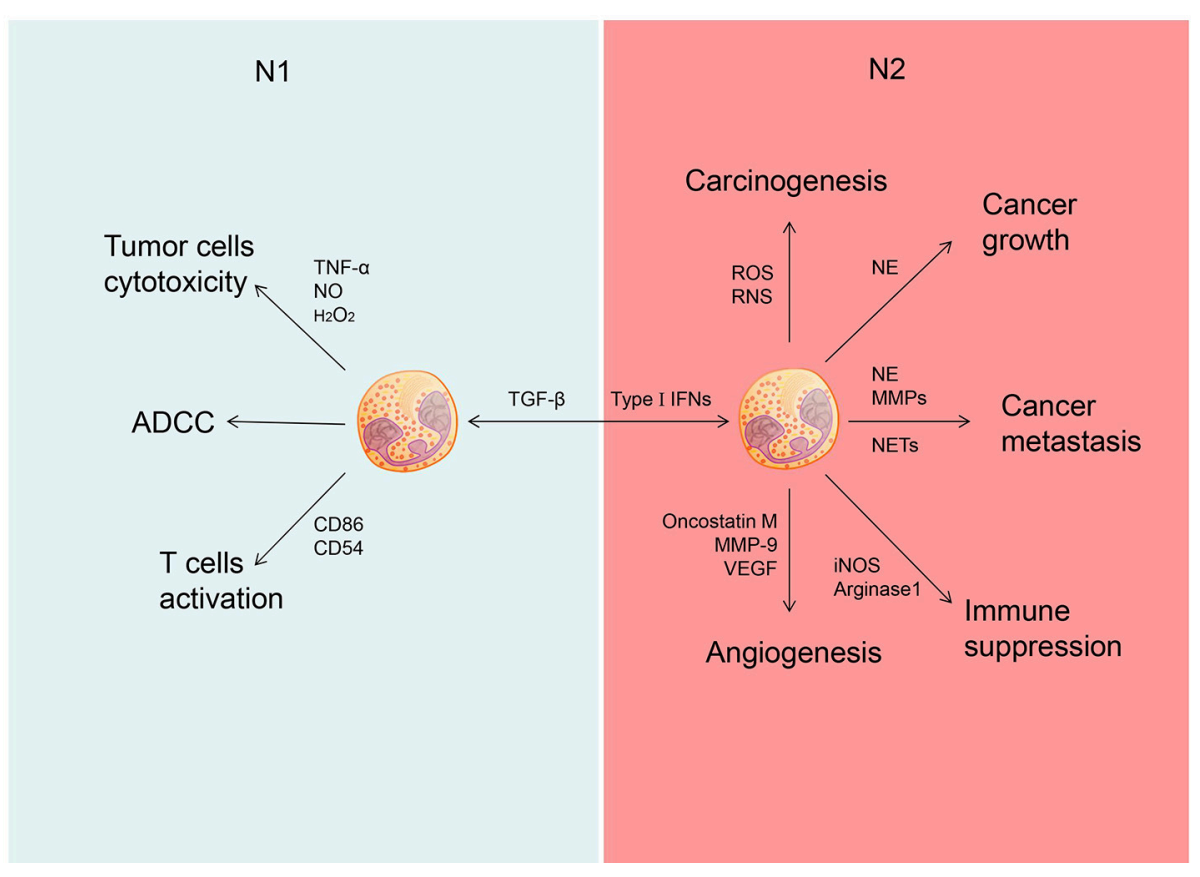

FIGURE 1 | Different role of N1 and N2 neutrophils in cancer. Neutrophils could be polarized into N1 phenotype under the induction of TGF-â and polarized into N2 phenotype under the induction of type I IFNs. N1 neutrophils could inhibit the development of cancer through tumor cells cytotoxicity, ADCC and activate T cells. N2 neutrophils could promote the development of cancer through promoting carcinogenesis, tumor growth, cancer metastasis, and cancer angiogenesis as well as suppress immunity.

that the ratio of neutrophil vs. lymphocyte may be a predictor for the progression of disability in patients with MS (12). Indeed, neutrophils from the peripheral blood of MS patients exhibit an inflammatory phenotype with increased degranulation, ROS production and NETs formation $(84,85)$. These neutrophils also show reduced apoptosis, which may contribute to the chronic inflammation and repeated relapse of MS (85). In addition, they can facilitate the disease pathogenesis by compromising bloodbrain-barrier $(\mathrm{BBB})$ and inducing oxidative stress via generation of $\operatorname{ROS}(60,61)$. BBB destruction by neutrophils is likely to result from increased production of myeloperoxidase (MPO) because inhibition of MPO restores integrity of $\mathrm{BBB}$ and ameliorates severity of disease (62).

Despite the potential harmful effect of neutrophils on MS, Gr$1^{\text {high }}$ neutrophils from central nervous system of EAE mice were shown to significantly suppress proliferation of myelin-reactive $\mathrm{T}$ cells, which is dependent on IFN- $\gamma$ production (86), suggesting the existence of a negative feedback pathway that limits the function of auto-reactive T cells in EAE. It is possible that Gr$1^{\text {high }}$ neutrophils maybe a subset of neutrophils with diseaseprotective activity in EAE. Given that G-MDSCs were previously characterized as $\mathrm{CD} 11 \mathrm{~b}^{+} \mathrm{Gr}-1^{\text {high }}$ immature cells in mice (87), these T cell-suppressive $\mathrm{Gr}-1^{+}$neutrophils observed in EAE mice may represent a subpopulation of G-MDSCs. In this context, it is reasonable to believe that the majority of neutrophils in EAE and MS are disease-promoting highly pro-inflammatory cells, and those with T cell-suppressive activity, e.g., Gr- $1^{+}$neutrophils or G-MDSCs, are unable to limit exacerbated inflammation to impact on disease progression. However, experimental evidence is required to support this possibility.

\section{Systemic Lupus Erythematosus}

An increase of a subpopulation of neutrophils, known as low density granulocytes (LDGs), was reported in systemic lupus erythematosus (SLE) many years ago (88). These cells display enhanced pro-inflammatory activity and increased synthesis of type I interferons (89). Intriguingly, normal high density neutrophils, upon incubation with the plasma from SLE patients, can be converted into LDGs. This decrease in their density may be caused by activation of normal neutrophils by certain soluble factors (e.g., immune complexes and complements), which is known to be associated with neutrophils degranulation and increased cell size (88). LDGs may promote the development of SLE by enhancing apoptosis of endothelial cells and impairing vascular repair. In this context, LDGs are also associated with an increased risk of vascular injury in SLE due to their endothelial cytotoxicity. LDGs also participate in the pathogenesis of SLE through increased NETs formation (63, 90). It was reported that the blockade of NETs formation alleviates the severity of disease (91). In this case, NETs not only can kill directly endothelia cells (63), but also enhance the production of inflammatory cytokines, including IL-1 $\beta$ and IL-18, via activation of the nucleotide-binding oligomerization domain-like receptors protein 3 (NLRP3) inflammasome in macrophages and promotion of IFN- $\alpha$ synthesis by plasmacytoid dendritic cells (pDC) (14). In addition, LDGs from SLE patients 
TABLE 1 | Neutrophils in autoimmune diseases.

\begin{tabular}{|c|c|c|c|c|}
\hline Diseases & Species & $\begin{array}{l}\text { Effector } \\
\text { molecules }\end{array}$ & Functions & References \\
\hline \multirow[t]{2}{*}{ MS } & Mouse & ROS & $\begin{array}{l}\text { Destructing } \\
\text { blood-brain-barrier }\end{array}$ & $(60,61)$ \\
\hline & Mouse & MPO & $\begin{array}{l}\text { Destructing } \\
\text { blood-brain-barrier }\end{array}$ & (62) \\
\hline \multirow[t]{2}{*}{ SLE } & Human & IFN-á & $\begin{array}{l}\text { Damaging vascular } \\
\text { endothelia }\end{array}$ & (13) \\
\hline & Human & NETs & $\begin{array}{l}\text { Killing endothelial cells } \\
\text { and activating } \\
\text { plasmacytoid dendritic } \\
\text { cells }\end{array}$ & $(14,63)$ \\
\hline \multirow[t]{6}{*}{ RA } & Mouse & PADI 4 & $\begin{array}{l}\text { Related to the } \\
\text { generation of } \\
\text { autoantibodies }\end{array}$ & (15) \\
\hline & Human & TNF & Attracting $T$ cells & (64) \\
\hline & Human & $\begin{array}{l}\text { B-lymphocyte } \\
\text { stimulator }\end{array}$ & Activating B cells & $(65,66)$ \\
\hline & Mouse & ROS & $\begin{array}{l}\text { Decreased ROS levels } \\
\text { is associated with } \\
\text { autoimmune and } \\
\text { chronic inflammation }\end{array}$ & $(67,68)$ \\
\hline & Human & MMPs & Destructing cartilage & $(69,70)$ \\
\hline & Mouse & RANKL & Resorbing bone & $(71)$ \\
\hline \multirow[t]{4}{*}{ T1D } & Human & $\mathrm{NE}$ & $\begin{array}{l}\text { Related to the } \\
\text { generation of â-cells } \\
\text { antigens autoantibodies }\end{array}$ & (72) \\
\hline & Human & Proteinase 3 & $\begin{array}{l}\text { Related to the } \\
\text { generation of â-cells } \\
\text { antigens autoantibodies }\end{array}$ & (72) \\
\hline & Mouse & ROS & $\begin{array}{l}\text { Destructing pancreatic } \\
\text { â-cells }\end{array}$ & (73) \\
\hline & Mouse & CRAMP & $\begin{array}{l}\text { Activating plasmacytoid } \\
\text { dendritic cells }\end{array}$ & (16) \\
\hline \multirow[t]{4}{*}{ IBD } & Mouse & ROS & $\begin{array}{l}\text { Killing bacteria and } \\
\text { damaging intestinal } \\
\text { mucusa }\end{array}$ & $(74,75)$ \\
\hline & Human & MMP-9 & $\begin{array}{l}\text { Degrading the } \\
\text { extracellular matrix and } \\
\text { vascular repairement }\end{array}$ & (76-78) \\
\hline & Human & NE & $\begin{array}{l}\text { Disrupting intestinal } \\
\text { barrier }\end{array}$ & $(79,80)$ \\
\hline & Mouse & IL-22 & $\begin{array}{l}\text { Repairing epithelial } \\
\text { integrity and resoling } \\
\text { colitis }\end{array}$ & (81) \\
\hline
\end{tabular}

produce higher levels of pro-inflammatory cytokines, such as IFN- $\alpha$ and TNF- $\alpha$, compared to autologous HDNs. However, the phagocytic capability of LDGs is significantly reduced (13), which is similar to the LDNs in cancer. Compared with LDNs from renal cell carcinoma patients, LDGs in SLE patients contains more percentage of immature neutrophils (10 vs. 40\%) (92). While LDNs in cancer express higher levels of CD11b and CD66b than HDNs, there is no difference in the levels of these activating markers between LDGs and HDGs in SLE $(13,92)$. Together, LDGs with a pro-inflammatory phenotype play a deleterious role in SLE development. While LDGs and LDNs in the circulations of cancer patients share similar phagocytic capability, LDNs are shown to have a distinct anti-inflammatory phenotype, suggesting that the density of neutrophils may not be a suitable marker to classify neutrophils subsets in different disease settings.

\section{Rheumatoid Arthritis}

It has been shown that neutrophil-to-lymphocyte ratio (NLR) correlates with the disease activity of rheumatoid arthritis (RA) (93-95). When combined with platelet-to-lymphocyte ratio, it can serve as a prognostic biomarker for RA (96). The detrimental role of neutrophils in RA may result from their association with the production of autoantibodies against citrullinated peptides, which is supported by finding that neutrophils in inflamed joints express peptidylarginine deiminase (PADI) 4 enzyme capable of catalyzing the citrullination of arginine (15). Neutrophils are involved in the recruitment and activation of $\mathrm{T}$ or $\mathrm{B}$ cells in RA. Neutrophils are an important source of TNF- $\alpha$, which can induce production of CCL18 to recruit T cells to inflamed sites (64). Neutrophils can also release B-lymphocyte stimulator to activate B cells in RA $(65,66)$. In addition, delayed apoptosis of neutrophils, due to activation of anti-apoptotic signals and inhibition of pro-apoptotic pathways by various cytokines, contributes to the perpetuation of inflammation and development of RA (15). Moreover, neutrophils participate in the destruction of cartilage by stimulating synoviocytes to release MMPs and activating osteoclast through receptor activator of nuclear factor-kB ligand (RANKL) signaling (69-71). In this context, they can promote bone adsorption and inhibit bone remodeling in RA.

While neutrophils from peripheral blood of patients with active synovitis generate less ROS than those from normal donors and arthritis patients at clinical remission, those in the synovial fluid (SF) of patients display increased ROS production (97). This phenomenon may be explained by the recruitment of primed neutrophils with high ROS production from the blood to the joint during disease progression. These findings indicate that there are at least two subsets of neutrophils with different capability of ROS production in RA. Reduced production of cellular ROS in circulating neutrophils increases the susceptibility of autoimmune diseases and the risk of chronic inflammation, whereas elevation of ROS in SF neutrophils may directly participate in injury of joints tissues $(67,68)$. Although specific phenotype of these neutrophils in RA has not been defined, clearly there exist two different subsets of neutrophils with different ROS production, both of which are deleterious to patients possibly through distinct mechanisms in circulation and SF.

\section{Type 1 Diabetes}

Type 1 diabetes (T1D) is an autoimmune disease with a characteristic of immune-mediated destruction of pancreatic- $\beta$ cells (98). T1D patients in prediabete stage or within 1 year after diagnosis show decreased number of neutrophils, and these cells increase to the normal level in those with T1D for more than 1 year (72). The reduction of neutrophils in T1D patients may be attributed, at least in part, to NETosis, that enhances 
formation of NETs and release of NE and proteinase 3 (PR3). The elevated levels of circulating NE and PR3 positively correlate to the increased seropositivity of autoantibodies reactive with $\beta$-cell antigen. NE and PR3 are also increased substantially even in autoantibody negative patients (72), suggesting that abnormal activity of neutrophil serine proteases may be involved in generation of autoantibodies and pathogenesis of disease at the early stage of T1D. However, a recent study reported that diabetic patients diagnosed within 3 years display decreased NE and PR3 levels (99). The discrepancies in the change of $\mathrm{NE}$ and PR3 may be caused by the differences in patient ages, disease severity, and stages. Therefore, the role of NETosis and its associated markers in the development of T1D need to be further investigated.

ROS, another important product of neutrophils, has also been reported to be elevated in patients with T1D (100), which may help initiate destruction of pancreatic $\beta$-cell (73). In addition, a number of cytokines produced by neutrophils, including IL1 , TNF- $\alpha$, and IFN- $\gamma$, can also participate in the initiation of pancreatic- $\beta$ cell destruction by stimulating production of toxic free radical or indirectly by inducing recruitment and activating of other immune cells (73). Since these cytokines are produced not only by neutrophils but also by other immune cell types and these immune cells are known to have pleiotropic and redundant effects, it is difficult to distinguish the role of individual cytokines in T1D. Other than their direct cytokine effect, the indirect effect of neutrophils on activation of other immune cells has also been documented. In non-obese diabetic (NOD) mice, DNA-anti-DNA IgG complexes stimulate formation of NETs and production of cathelicidin-related antimicrobial peptide (CRAMP). Subsequent activation of $\mathrm{pDC}$ and concurrent release of IFN- $\alpha$ promotes $\mathrm{T}$ cell-mediated autoimmune response at the early stage of T1D (16). Therefore, neutrophils in T1D appear to contribute to immune pathology by facilitating auto-antibody-mediated $\beta$-cell destruction and by enhancing inflammation. Given the heterogeneity of this cell population, it is intriguing to address the question as to whether antiinflammatory and disease-protective neutrophils are present in T1D.

\section{Inflammatory Bowel Diseases}

Inflammatory bowel diseases (IBD), including Crohn's disease (CD), and ulcerative colitis (UC), are characterized by chronic, relapsing inflammation in gastrointestinal tract, which are resulted from dysregulation of immune responses at intestinal mucosa (101). The role of neutrophils in the pathogenesis of IBD remains controversial. The neutrophil count in patients with IBD is significantly increased when compared to that in normal individuals (102). The number of infiltrating neutrophils is associated with the severity of UC (103). Patients with active UC often have a higher neutrophil-to-lymphocyte ratio than normal controls (104). These results suggest that neutrophils recruited to the lesion may be deleterious to the development of UC. Neutrophils can also be involved in regulation of disease development via MMP-9-mediated degradation of ECM. MMP-9 deficiency alleviates an inflammatory response and intestinal injury in DSS-induced IBD mice (76), and the inhibition of MMP-9 reduces disease severity (77). In addition, increased activity of NE in UC patients support potential involvement of NE in the pathogenesis of IBD (105). Overreactive NE can disrupt intestinal barrier by degrading Ecadherins or zonula occludens-1 (79), and inhibit mucosal repair by suppressing proliferation of intestinal epithelial cells (80). However, defect of neutrophil recruitment contributes to the inefficient bacteria clearance and chronic inflammation in CD (106-108). Neutrophils from peripheral blood of CD patients produce increased ROS (109), which exhibit important antimicrobial properties known to protect host against microbial infections (74). However, over produced ROS can cause intestinal mucosal damage, thereby increasing mucosal permeability due to the degradation of polyunsaturated acids in the membrane of intestinal epithelial cells (75). Therefore, the suppression of ROS production, such as anti-TNF- $\alpha$ (infliximab) therapy, will be beneficial for IBD patients (110).

A recent study reported that $\mathrm{CD} 177^{+}$neutrophils exhibit a protective role against IBD. CD177 is a surface marker expressed exclusively on human neutrophils (111) and 45-60\% of neutrophils in periphery blood of healthy individuals are $\mathrm{CD} 177$ positive (112). The $\mathrm{CD}_{177^{+}}$neutrophils have enhanced antimicrobial activity, which is associated with increased production of ROS, MPO, NETs as well as bactericidal peptides (17). Compared to $\mathrm{CD}_{177^{-}}$counterparts, $\mathrm{CD} 177^{+}$neutrophils display lower levels of pro-inflammatory cytokines, such as IFN$\gamma$, IL-6, and IL-17A, but higher level of IL-22 (17), which promote restoration of epithelial integrity and resolution of colitis. CD177 knockout mice develop more severe colitis induced by DSS and compromised intestinal barrier integrity than wild-type mice (81). CD177 deficiency may impair accumulation of neutrophil to infected sites at the early stage of disease and therefore cause increased intestinal mucosa destruction (113). This association of neutrophils accumulation at the inflamed sites with CD177dependent protection from colitis induced by DSS suggests these $\mathrm{CD} 177^{+}$neutrophils are anti-inflammatory and IBD-protective and appear to be distinct from pro-inflammatory LDGs found in SLE.

\section{CONCLUSION AND FUTURE PERSPECTIVE}

Neutrophils demonstrate multifaceted functions in cancers and autoimmune diseases, which are determined by their context dependent production of different effector molecules. These cells are generally divided into N1/N2 subsets in tumor site or alternatively HDNs/LDNs in peripheral blood of cancer patients. Although HDNs and LDNs can be distinguished based on their differences in density, currently there are no validated surface markers to phenotypically identify $\mathrm{N} 1$ and N2 neutrophils. However, LDGs in SLE and LDNs in cancer are functionally distinct, which raise a question of using density for neutrophils classification. It is clear that there are at least two subsets of neutrophils defined as immune-stimulatory/-suppressive or pro-/anti-inflammatory. These two subsets of neutrophils are highly plastic and can be skewed toward either direction, which is 
exemplified by type I interferon-driven polarization of immunesuppressive neutrophils into ones with an immunostimulatory phenotype.

Currently, neutrophils in autoimmune diseases do not have a clear classification. In most cases, they display a proinflammatory phenotype and promote the disease pathogenesis through multiple mechanisms (secretion of inflammatory molecules, activation of other immune cells, facilitate production of auto-antibodies). However, anti-inflammatory and diseaseprotective neutrophils have been identified in autoimmune conditions, including Gr-1 $1^{\text {high }}$ neutrophils in EAE and CD177 ${ }^{+}$ neutrophils in IBD, which supports the functional complexity of these heterogeneous immune cells. Although the tumorpromoting activity of $\mathrm{N} 1$ neutrophils has been well documented, little studies have been performed to target these cells for cancer therapy so far. A better understanding of these antiinflammatory and disease-protective neutrophils associated with some autoimmune pathology may provide a potential new target for the treatment of these autoimmune disorders.

Understanding of the role of neutrophils is far from being complete. In the future, more research is needed to address the following questions. What are proper phenotypic markers to distinguish immuno-stimulatory/-suppressive or pro-/anti-inflammatory neutrophils? Are anti-inflammatory or immunosuppressive neutrophils exist broadly in all autoimmune diseases or are only present in specific autoimmune setting? Can TGF- $\beta$ and type I interferons induce reciprocal polarization

\section{REFERENCES}

1. Sionov RV, Fridlender ZG, Granot Z. The multifaceted roles neutrophils play in the tumor microenvironment. Cancer Microenviron. (2015) 8:125-58. doi: $10.1007 / \mathrm{s} 12307-014-0147-5$

2. Mayadas TN, Cullere X, Lowell CA. The multifaceted functions of neutrophils. Annu Rev Pathol. (2014) 9:181-218. doi: 10.1146/annurev-pathol-020712-164023

3. Amulic B, Cazalet C, Hayes GL, Metzler KD, Zychlinsky A. Neutrophil function: from mechanisms to disease. Ann Rev Immunol. (2012) 30:459-89. doi: 10.1146/annurev-immunol-020711-074942

4. Brandau S, Moses K, Lang S. The kinship of neutrophils and granulocytic myeloid-derived suppressor cells in cancer: cousins, siblings or twins? Semin Cancer Biol. (2013) 23:171-82. doi: 10.1016/j.semcancer.2013.02.007

5. Piccard H, Muschel RJ, Opdenakker G. On the dual roles and polarized phenotypes of neutrophils in tumor development and progression. Critic Rev Oncol/Hematol. (2012) 82:296-309. doi: 10.1016/j.critrevonc.2011.06.004

6. Brandau S, Dumitru CA, Lang S. Protumor and antitumor functions of neutrophil granulocytes. Semin Immunopathol. (2013) 35:163-76. doi: 10.1007/s00281-012-0344-6

7. Kobayashi Y. Neutrophil biology: an update. EXCLI J. (2015) 14:220-7. doi: 10.17179/excli2015-102

8. Hu W, Li X, Zhang C, Yang Y, Jiang J, Wu C. Tumor-associated macrophages in cancers. Clin Trans Oncol. (2016) 18:251-8. doi: 10.1007/s12094-015-1373-0

9. Sica A, Larghi P, Mancino A, Rubino L, Porta C, Totaro MG, et al. Macrophage polarization in tumour progression. Semin Cancer Biol. (2008) 18:349-55. doi: 10.1016/j.semcancer.2008.03.004

10. Sagiv JY, Voels S, Granot Z. Isolation and characterization of low- vs. highdensity neutrophils in cancer. In: Ursini-Siegel J, Beauchemin N, editors. The Tumor Microenvironment: Methods and Protocols. New York, NY: Springer. (2016) p. 179-93. doi: 10.1007/978-1-4939-3801-8_13 of pro- or anti-inflammatory neutrophils in the context of autoimmune diseases? What is the relationship between anti-inflammatory neutrophils in autoimmune diseases and immunosuppressive neutrophils in cancer? Can they be classified into the same subpopulation? Answers to these questions will provide important insights into the development and function of neutrophils in cancer and autoimmune diseases, which may lead to development of novel approaches to disease intervention.

\section{AUTHOR CONTRIBUTIONS}

All authors listed have made a substantial, direct and intellectual contribution to the work, and approved it for publication. XW and LQ wrote the manuscript. HY, ZL, and X-YW revised the manuscript.

\section{FUNDING}

The research was supported by grant from NSFC (81671592) and the Science and Technology Department of Jilin Province (20180101110JC).

\section{ACKNOWLEDGMENTS}

We thank Professor Yun Dai from Lab of Cancer Precision Medicine for very helpful comments and suggestions on this manuscript.
11. Sagiv Jitka Y, Michaeli J, Assi S, Mishalian I, Kisos H, Levy L, et al. Phenotypic diversity and plasticity in circulating neutrophil subpopulations in cancer. Cell Rep. (2015) 10:562-73. doi: 10.1016/j.celrep.2014.12.039

12. Demirci S, Demirci S, Kutluhan S, Koyuncuoglu HR, Yurekli VA. The clinical significance of the neutrophil-to-lymphocyte ratio in multiple sclerosis. Int J Neurosci. (2016) 126:700-6. doi: 10.3109/00207454.2015.1050492

13. Denny MF, Yalavarthi S, Zhao W, Thacker SG, Anderson M, Sandy AR, et al. A distinct subset of proinflammatory neutrophils isolated from patients with systemic lupus erythematosus induces vascular damage and synthesizes type I IFNs. J Immunol. (2010) 184:3284-97. doi: 10.4049/jimmunol.09 02199

14. Smith CK, Kaplan MJ. The role of neutrophils in the pathogenesis of systemic lupus erythematosus. Curr Opin Rheumatol. (2015) 27:448-53. doi: 10.1097/BOR.0000000000000197

15. Cascão R, Rosário HS, Souto-Carneiro MM, Fonseca JE. Neutrophils in rheumatoid arthritis: more than simple final effectors. Autoimmun Rev. (2010) 9:531-5. doi: 10.1016/j.autrev.2009.12.013

16. Battaglia M. Neutrophils and type 1 autoimmune diabetes. Curr Opin Hematol. (2014) 21:8-15. doi: 10.1097/MOH.0000000000000008

17. Zhou G, Yu L, Fang L, Yang W, Yu T, Miao Y, et al. CD177+ neutrophils as functionally activated neutrophils negatively regulate IBD. Gut (2017) 67:1052-63. doi: 10.1136/gutjnl-2016-313535

18. Mills C. M1 and M2 Macrophages: oracles of health and disease. Crit Rev Immunol. (2012) 32:463-88. doi: 10.1615/CritRevImmunol.v32.i6.10

19. Swiecki M, Colonna M. The multifaceted biology of plasmacytoid dendritic cells. Nat Rev Immunol. (2015) 15:471-85. doi: 10.1038/nri3865

20. Reizis B, Bunin A, Ghosh HS, Lewis KL, Sisirak V. Plasmacytoid dendritic cells: recent progress and open questions. Annu Rev Immunol. (2011) 29:163-83. doi: 10.1146/annurev-immunol-031210-101345

21. Zhang Y, Zhang Y, Gu W, Sun B. TH1/TH2 cell differentiation and molecular signals. Adv Exp Med Biol. (2014) 841:15-44. doi: 10.1007/978-94-017-9487-9_2 
22. Murphy PM. Neutrophil receptors for interleukin-8 and related CXC chemokines. Semin Hematol. (1997) 34:311-8.

23. Viola A, Sarukhan A, Bronte V, Molon B. The pros and cons of chemokines in tumor immunology. Trends Immunol. (2012) 33:496-504. doi: 10.1016/j.it.2012.05.007

24. Pillay J, den Braber I, Vrisekoop N, Kwast LM, de Boer RJ, Borghans JA, et al. In vivo labeling with $2 \mathrm{H} 2 \mathrm{O}$ reveals a human neutrophil lifespan of 5.4 days. Blood (2010) 116:625-7. doi: 10.1182/blood-2010-01-259028

25. Dancey JT, Deubelbeiss KA, Harker LA, Finch CA. Neutrophil kinetics in man. J Clin Invest. (1976) 58:705-15. doi: 10.1172/JCI108517

26. Fridlender ZG, Albelda SM. Tumor-associated neutrophils: friend or foe? Carcinogenesis (2012) 33:949-55. doi: 10.1093/carcin/bgs123

27. Coffelt SB, Wellenstein MD, de Visser KE. Neutrophils in cancer: neutral no more. Nat Rev Cancer (2016) 16:431-46. doi: 10.1038/nrc.2016.52

28. Wislez M, Rabbe N, Marchal J, Milleron B, Crestani B, Mayaud C, et al. Hepatocyte growth factor production by neutrophils infiltrating bronchioloalveolar subtype pulmonary adenocarcinoma. Role Tumor Progr Death (2003) 63:1405-12.

29. Powell DR, Huttenlocher A. Neutrophils in the tumor microenvironment. Trends Immunol. (2016) 37:41-52. doi: 10.1016/j.it.2015.11.008

30. Dumitru CA, Lang S, Brandau S. Modulation of neutrophil granulocytes in the tumor microenvironment: mechanisms and consequences for tumor progression. Semin Cancer Biol. (2013) 23:141-8. doi: 10.1016/j.semcancer.2013.02.005

31. Mishalian I, Bayuh R, Levy L, Zolotarov L, Michaeli J, Fridlender ZG. Tumor-associated neutrophils (TAN) develop pro-tumorigenic properties during tumor progression. Cancer Immunol Immunother. (2013) 62:174556. doi: 10.1007/s00262-013-1476-9

32. Fridlender ZG, Sun J, Kim S, Kapoor V, Cheng G, Ling L, et al. Polarization of tumor-associated neutrophil phenotype by TGF-â: "N1" versus "N2" TAN. Cancer Cell (2009) 16:183-94. doi: 10.1016/j.ccr.2009.06.017

33. Pylaeva E, Lang S, Jablonska J. The essential role of type I interferons in differentiation and activation of tumor-associated neutrophils. Front Immunol. (2016) 7:629. doi: 10.3389/fimmu.2016.00629

34. Shrestha S, Noh JM, Kim S-Y, Ham H-Y, Kim Y-J, Yun Y-J, et al. Angiotensin converting enzyme inhibitors and angiotensin II receptor antagonist attenuate tumor growth via polarization of neutrophils toward an antitumor phenotype. OncoImmunology (2016) 5:e1067744. doi: 10.1080/2162402X.2015.1067744

35. Boyum A. Isolation of mononuclear cells and granulocytes from human blood. isolation of monuclear cells by one centrifugation, and of granulocytes by combining centrifugation and sedimentation at $1 \mathrm{~g}$. Scand J Clin Lab Invest. (1968) 97(Suppl.):77-89.

36. Mishalian I, Granot Z, Fridlender ZG. The diversity of circulating neutrophils in cancer. Immunobiology (2017) 222:82-8. doi: 10.1016/j.imbio.2016.02.001

37. Pillay J, Tak T, Kamp VM, Koenderman L. Immune suppression by neutrophils and granulocytic myeloid-derived suppressor cells: similarities and differences. Cell Mol Life Sci. (2013) 70:3813-27. doi: 10.1007/s00018-013-1286-4

38. Gabrilovich DI, Nagaraj S. Myeloid-derived suppressor cells as regulators of the immune system. Nat Rev Immunol. (2009) 9:162-74. doi: $10.1038 /$ nri2506

39. Greifenberg V, Ribechini E, Rößner S, Lutz MB. Myeloid-derived suppressor cell activation by combined LPS and IFN- $\gamma$ treatment impairs DC development. Eur J Immunol. (2009) 39:2865-76. doi: 10.1002/eji.200939486

40. Zilio S, Serafini P. Neutrophils and granulocytic MDSC: the janus god of cancer immunotherapy. Vaccines (2016) 4:31. doi: 10.3390/vaccines4030031

41. van Egmond M, Bakema JE. Neutrophils as effector cells for antibodybased immunotherapy of cancer. Semin Cancer Biol. (2013) 23:190-9. doi: 10.1016/j.semcancer.2012.12.002

42. Eruslanov EB, Bhojnagarwala PS, Quatromoni JG, Stephen TL, Ranganathan A, Deshpande C, et al. Tumor-associated neutrophils stimulate $\mathrm{T}$ cell responses in early-stage human lung cancer. J Clin Invest. (2014) 124:546680. doi: 10.1172/JCI77053

43. Eruslanov EB. Phenotype and function of tumor-associated neutrophils and their subsets in early-stage human lung cancer. Cancer Immunol Immunother. (2017) 2017:1-10. doi: 10.1007/s00262-017-1976-0
44. Ho A-S, Chen C-H, Cheng C-C, Wang C-C, Lin H-C, Luo T$\mathrm{Y}$, et al. Neutrophil elastase as a diagnostic marker and therapeutic target in colorectal cancers. Oncotarget (2014) 5:2. doi: 10.18632/ oncotarget.1631

45. Kim JH, Lee JY, Kim HK, Lee JW, Jung SG, Jung K, et al. Prognostic significance of the neutrophil-to-lymphocyte ratio and platelet-tolymphocyte ratio in patients with stage III and IVcolorectal cancer. World J Gastroenterol. (2017) 23:505-15. doi: 10.3748/wjg.v23.i3.505

46. Bambury RM, Teo MY, Power DG, Yusuf A, Murray S, Battley JE, et al. The association of pre-treatment neutrophil to lymphocyte ratio with overall survival in patients with glioblastoma multiforme. J Neuro-Oncol. (2013) 114:149-54. doi: 10.1007/s11060-013-1164-9

47. Arai K, Fukumoto T, Kido M, Tanaka M, Kuramitsu K, Kinoshita H, et al. Preoperative neutrophil-to-lymphocyte ratio as a predictor of survival after reductive surgery plus percutaneous isolated hepatic perfusion for hepatocellular carcinoma: a retrospective analysis. Surgery Today (2017) 47:385-92. doi: 10.1007/s00595-016-1384-7

48. Park YH, Ku JH, Kwak C, Kim HH. Post-treatment neutrophil-tolymphocyte ratio in predicting prognosis in patients with metastatic clear cell renal cell carcinoma receiving sunitinib as first line therapy. SpringerPlus (2014) 3:243. doi: 10.1186/2193-1801-3-243

49. Ferrucci PF, Ascierto PA, Pigozzo J, Del Vecchio M, Maio M, Antonini Cappellini GC, et al. Baseline neutrophils and derived neutrophil-to-lymphocyte ratio: prognostic relevance in metastatic melanoma patients receiving ipilimumab. Ann Oncol. (2016) 27:732-8. doi: 10.1093/annonc/mdw016

50. Camacho Limas CP, Gerson Cwilich R, Góngora Jurado MdÁ, López Naveda M. Asociación del índice neutrófilo-linfocito y el estadio clínico en el diagnóstico inicial de melanoma. Gaceta Mexicana Oncol. (2016) 15:268-77. doi: 10.1016/j.gamo.2016.08.002

51. Glazer ES, Rashid OM, Pimiento JM, Hodul PJ, Malafa MP. Increased neutrophil-to-lymphocyte ratio after neoadjuvant therapy is associated with worse survival after resection of borderline resectable pancreatic ductal adenocarcinoma. Surgery (2016) 160:1288-93. doi: 10.1016/j.surg.2016.04.039

52. Kim DY, Kim IS, Park SG, Kim H, Choi YJ, Seol YM. Prognostic value of posttreatment neutrophil-lymphocyte ratio in head and neck squamous cell carcinoma treated by chemoradiotherapy. Auris Nasus Larynx (2017) 44:199-204. doi: 10.1016/j.anl.2016.05.013

53. Finisguerra V, Di Conza G, Di Matteo M, Serneels J, Costa S, Thompson AAR, et al. MET is required for the recruitment of anti-tumoural neutrophils. Nature (2015) 522:349-53. doi: 10.1038/nature14407

54. Queen MM, Ryan RE, Holzer RG, Keller-Peck CR, Jorcyk CL. Breast cancer cells stimulate neutrophils to produce oncostatin $\mathrm{M}$ : potential implications for tumor progression. Cancer Res. (2005) 65:8896-904. doi: 10.1158/0008-5472.CAN-05-1734

55. Lauber S, Wong S, Cutz JC, Tanaka M, Barra N, Lhotak S, et al. Novel function of Oncostatin $\mathrm{M}$ as a potent tumour-promoting agent in lung. Int J Cancer (2015) 136:831-43. doi: 10.1002/ijc.29055

56. Nozawa H, Chiu C, Hanahan D. Infiltrating neutrophils mediate the initial angiogenic switch in a mouse model of multistage carcinogenesis. Proc Natl Acad Sci USA. (2006) 103:12493-8. doi: 10.1073/pnas.06018 07103

57. Rotondo R, Barisione G, Mastracci L, Grossi F, Orengo AM, Costa R, et al. IL-8 induces exocytosis of arginase 1 by neutrophil polymorphonuclears in nonsmall cell lung cancer. Int J Cancer (2009) 125:887-93. doi: $10.1002 /$ ijc. 24448

58. Cools-Lartigue J, Spicer J, Najmeh S, Ferri L. Neutrophil extracellular traps in cancer progression. Cell Mol Life Sci. (2014) 71:4179-94. doi: 10.1007/s00018-014-1683-3

59. Tillack K, Breiden P, Martin R, Sospedra M. T lymphocyte priming by neutrophil extracellular traps links innate and adaptive immune responses. J Immunol. (2012) 188:3150-9. doi: 10.4049/jimmunol.11 03414

60. Miller N, Wang J, Tan Y, Dittel B. Anti-inflammatory mechanisms of IFN- $\gamma$ studied in experimental autoimmune encephalomyelitis reveal neutrophils as a potential target in multiple sclerosis. Front Neurosci. (2015) 9:287. doi: $10.3389 /$ fnins.2015.00287 
61. Gilgun-Sherki Y, Melamed E, Offen D. The role of oxidative stress in the pathogenesis of multiple sclerosis: the need for effective antioxidant therapy. J Neurol. (2004) 251:261-8. doi: 10.1007/s00415-004-0348-9

62. Zhang H, Ray A, Miller NM, Hartwig D, Pritchard KA, Dittel BN. Inhibition of myeloperoxidase at the peak of experimental autoimmune encephalomyelitis restores blood-brain barrier integrity and ameliorates disease severity. I Neurochem. (2016) 136:826-36. doi: 10.1111/jnc. 13426

63. Villanueva E, Yalavarthi S, Berthier CC, Hodgin JB, Khandpur R, Lin AM, et al. Netting neutrophils induce endothelial damage, infiltrate tissues, and expose immunostimulatory molecules in systemic lupus erythematosus. $J$ Immunol. (2011) 187:538-52. doi: 10.4049/jimmunol.1100450

64. Auer J, Blass M, Schulze-Koops H, Russwurm S, Nagel T, Kalden JR, et al. Expression and regulation of CCL18 in synovial fluid neutrophils of patients with rheumatoid arthritis. Arthritis Res Ther. (2007) 9:R94. doi: 10.1186/ar2294

65. Kasama T, Kobayashi K, Yajima N, Shiozawa F, Yoda Y, Takeuchi HT, et al. Expression of vascular endothelial growth factor by synovial fluid neutrophils in rheumatoid arthritis (RA). Clin Exp Immunol. (2000) 121:533-8. doi: 10.1046/j.1365-2249.2000.01272.x

66. Cheema GS, Roschke V, Hilbert DM, Stohl W. Elevated serum B lymphocyte stimulator levels in patients with systemic immune-based rheumatic diseases. Arthritis Rheum. (2001) 44:1313-9. doi: 10.1002/15290131(200106)44:6<1313::AID-ART223>3.0.CO;2-S

67. Bjorkman L, Dahlgren C, Karlsson A, Brown KL, Bylund J. Phagocytederived reactive oxygen species as suppressors of inflammatory disease. Arthritis Rheum. (2008) 58:2931-5. doi: 10.1002/art. 23941

68. Bylund J, MacDonald KL, Brown KL, Mydel P, Collins LV, Hancock RE, et al. Enhanced inflammatory responses of chronic granulomatous disease leukocytes involve ROS-independent activation of NF-kappa B. Eur J Immunol. (2007) 37:1087-96. doi: 10.1002/eji.200636651

69. Wang C-H, Dai J-Y, Wang L, Jia J-F, Zheng Z-H, Ding J, et al. Expression of CD147 (EMMPRIN) on neutrophils in rheumatoid arthritis enhances chemotaxis, matrix metalloproteinase production and invasiveness of synoviocytes. J Cell Mol Med. (2011) 15:850-60. doi: 10.1111/j.1582-4934.2010.01084.x

70. Moore AR, Iwamura H, Larbre JP, Scott DL, Willoughby DA. Cartilage degradation by polymorphonuclear leucocytes: in vitro assessment of the pathogenic mechanisms. Ann Rheum Dis. (1993) 52:27-31. doi: 10.1136/ard.52.1.27

71. Hofbauer LC, Heufelder AE. The role of osteoprotegerin and receptor activator of nuclear factor kappaB ligand in the pathogenesis and treatment of rheumatoid arthritis. Arthritis Rheum. (2001) 44:253-9. doi: 10.1002/ 1529-0131(200102)44:2<253::AID-ANR41>3.0.CO;2-S

72. Wang Y, Xiao Y, Zhong L, Ye D, Zhang J, Tu Y, et al. Increased neutrophil elastase and proteinase 3 and augmented NETosis are closely associated with beta-cell autoimmunity in patients with type 1 diabetes. Diabetes (2014) 63:4239-48. doi: 10.2337/db14-0480

73. Huang J, Xiao Y, Xu A, Zhou Z. Neutrophils in type 1 diabetes. J Diabetes Invest. (2016) 7:652-63. doi: 10.1111/jdi.12469

74. Mouzaoui S, Djerdjouri B, Makhezer N, Kroviarski Y, El-Benna J, Dang PM. Tumor necrosis factor-alpha-induced colitis increases NADPH oxidase 1 expression, oxidative stress, and neutrophil recruitment in the colon: preventive effect of apocynin. Mediators Inflamm. (2014) 2014:312484. doi: 10.1155/2014/312484

75. Naito Y, Takagi T, Yoshikawa T. Neutrophil-dependent oxidative stress in ulcerative colitis. J Clin Biochem Nutr. (2007) 41:18-26. doi: $10.3164 /$ jcbn. 2007003

76. Nighot P, Al-Sadi R, Rawat M, Guo S, Watterson DM, Ma T. Matrix metalloproteinase 9-induced increase in intestinal epithelial tight junction permeability contributes to the severity of experimental DSS colitis. Am J Physiol Gastrointest Liver Physiol. (2015) 309:G988-97. doi: 10.1152/ajpgi.00256.2015

77. Marshall DC, Lyman SK, McCauley S, Kovalenko M, Spangler R, Liu C, et al. Selective allosteric inhibition of MMP9 is efficacious in preclinical models of ulcerative colitis and colorectal cancer. PLOS ONE (2015) 10:e0127063. doi: 10.1371 /journal.pone. 0127063
78. Ardi VC, Kupriyanova TA, Deryugina EI, Quigley JP. Human neutrophils uniquely release TIMP-free MMP-9 to provide a potent catalytic stimulator of angiogenesis. Proc Natl Acad Sci USA. (2007) 104:20262-7. doi: 10.1073/pnas.0706438104

79. Ginzberg HH, Cherapanov V, Dong Q, Cantin A, McCulloch CA, Shannon PT, et al. Neutrophil-mediated epithelial injury during transmigration: role of elastase. Am J Physiol Gastrointest Liver Physiol. (2001) 281:G705-17. doi: 10.1152/ajpgi.2001.281.3.G705

80. Kuno Y, Ina K, Nishiwaki T, Tsuzuki T, Shimada M, Imada A, et al. Possible involvement of neutrophil elastase in impaired mucosal repair in patients with ulcerative colitis. J Gastroenterol. (2002) 37(Suppl. 14):22-32. doi: 10.1007/BF03326409

81. Zindl CL, Lai JF, Lee YK, Maynard CL, Harbour SN, Ouyang W, et al. IL22-producing neutrophils contribute to antimicrobial defense and restitution of colonic epithelial integrity during colitis. Proc Natl Acad Sci USA. (2013) 110:12768-73. doi: 10.1073/pnas.1300318110

82. Wu F, Cao W, Yang Y, Liu A. Extensive infiltration of neutrophils in the acute phase of experimental autoimmune encephalomyelitis in C57BL/6 mice. Histochem Cell Biol. (2010) 133:313-22. doi: 10.1007/s00418-009-0673-2

83. Casserly CS, Nantes JC, Whittaker Hawkins RF, Vallières L. Neutrophil perversion in demyelinating autoimmune diseases: Mechanisms to medicine. Autoimmun Rev. (2017) 16:294-307 doi: 10.1016/j.autrev.2017.01.013

84. Hertwig L, Pache F, Romero-Suarez S, Stürner KH, Borisow N, Behrens $\mathrm{J}$, et al. Distinct functionality of neutrophils in multiple sclerosis and neuromyelitis optica. Multiple Sclerosis J. (2015) 22:160-73. doi: $10.1177 / 1352458515586084$

85. Naegele M, Tillack K, Reinhardt S, Schippling S, Martin R, Sospedra M. Neutrophils in multiple sclerosis are characterized by a primed phenotype. J Neuroimmunol. (2012) 242:60-71. doi: 10.1016/j.jneuroim.2011.11.009

86. Zehntner SP, Brickman C, Bourbonnière L, Remington L, Caruso M, Owens T. Neutrophils that infiltrate the central nervous system regulate $\mathrm{T}$ cell responses. J Immunol. (2005) 174:5124-31. doi: 10.4049/jimmunol.174.8.5124

87. Youn J-I, Nagaraj S, Collazo M, Gabrilovich DI. Subsets of myeloid-derived suppressor cells in tumor-bearing mice. J Immunol. (2008) 181:5791-802. doi: 10.4049/jimmunol.181.8.5791

88. Hacbarth E, Kajdacsy-Balla A. Low density neutrophils in patients with systemic lupus erythematosus, rheumatoid arthritis, and acute rheumatic fever. Arthritis Rheum. (1986) 29:1334-42. doi: 10.1002/art.1780291105

89. Carmona-Rivera C, Kaplan MJ. Low-density granulocytes: a distinct class of neutrophils in systemic autoimmunity. Semin Immunopathol. (2013) 35:455-63 doi: 10.1007/s00281-013-0375-7

90. Knight JS, Kaplan MJ. Lupus neutrophils: 'NET' gain in understanding lupus pathogenesis. Curr Opin Rheumatol. (2012) 24:441-50. doi: 10.1097/BOR.0b013e3283546703

91. Knight JS, Zhao W, Luo W, Subramanian VX, O’Dell AA, Yalavarthi S, et al. Peptidylarginine deiminase inhibition is immunomodulatory and vasculoprotective in murine lupus. J Clin Invest. (2013) 123:2981-93. doi: 10.1172/JCI67390

92. Rodriguez PC, Ernstoff MS, Hernandez C, Atkins M, Zabaleta J, Sierra R, et al. Arginase I-producing myeloid-derived suppressor cells in renal cell carcinoma are a subpopulation of activated granulocytes. Cancer Res. (2009) 69:1553-60. doi: 10.1158/0008-5472.CAN-08-1921

93. Mercan R, Bitik B, Tufan A, Bozbulut UB, Atas N, Ozturk MA, et al. The association between neutrophil/lymphocyte ratio and disease activity in rheumatoid arthritis and ankylosing spondylitis. J Clin Lab Analysis (2016) 30:597-601. doi: 10.1002/jcla.21908

94. Fu HT, Qin BD, Hu ZD, Ma N, Yang M, Wei TT, et al. Neutrophiland platelet-to-lymphocyte ratios are correlated with disease activity in rheumatoid arthritis. Clin Lab. (2015) 61:269-73. doi: 10.7754/Clin.Lab.2014.140927

95. Koiwa M, Goto S, Takahashi K, Kamada T, Takai S, Nakamura H. Neutrophil/lymphocyte ratio in patients with rheumatoid arthritis treated with biological agents. J Nippon Med Sch. (2016) 83:118-24. doi: 10.1272/jnms.83.118

96. Zhang YY, Yin YM, Kuai SG, Shan ZB, Pei H, Wang J. Combination of neutrophil to lymphocyte ratio and platelet to lymphocyte ratio as diagnostic 
biomarker for rheumatoid arthritis. Int J Clin Exp Med. (2016) 9:22076-81. doi: 10.1136/annrheumdis-2014-205365

97. Cedergren J, Forslund T, Sundqvist T, Skogh T. Intracellular oxidative activation in synovial fluid neutrophils from patients with rheumatoid arthritis but not from other arthritis patients. J Rheumatol. (2007) 34:216270 .

98. Pietropaolo M, Towns R, Eisenbarth GS. Humoral autoimmunity in type 1 diabetes: prediction, significance, and detection of distinct disease subtypes. Cold Spring Harbor Perspect Med. (2012) 2:10. doi: 10.1101/cshperspect.a012831

99. Qin J, Fu S, Speake C, Greenbaum CJ, Odegard JM. NETosisassociated serum biomarkers are reduced in type 1 diabetes in association with neutrophil count. Clin Exp Immunol. (2016) 184:318-22. doi: $10.1111 /$ cei. 12783

100. Wierusz-Wysocka B, Wysocki H, Siekierka H, Wykretowicz A, Szczepanik A, Klimas R. Evidence of polymorphonuclear neutrophils (PMN) activation in patients with insulin-dependent diabetes mellitus. J Leukoc Biol. (1987) 42:519-23. doi: $10.1002 / \mathrm{jlb} .42 .5 .519$

101. Corridoni D, Arseneau KO, Cominelli F. Inflammatory bowel disease. Immunol Lett. (2014) 161:231-5. doi: 10.1016/j.imlet.2014.04.004

102. Hanai H, Takeuchi K, Iida T, Kashiwagi N, Saniabadi AR, Matsushita I, et al. Relationship between fecal calprotectin, intestinal inflammation, and peripheral blood neutrophils in patients with active ulcerative colitis. Digest Dis Sci. (2004) 49:1438-43. doi: 10.1023/B:DDAS.0000042243. 47279.87

103. Bressenot A, Salleron J, Bastien C, Danese S, Boulagnon-Rombi C, PeyrinBiroulet L. Comparing histological activity indexes in UC. Gut (2015) 64:1412-8. doi: 10.1136/gutjnl-2014-307477

104. Demir AK, Demirtas A, Kaya SU, Tastan I, Butun I, Sagcan M, et al. The relationship between the neutrophil-lymphocyte ratio and disease activity in patients with ulcerative colitis. Kaohsiung J Med Sci. (2015) 31:585-90. doi: 10.1016/j.kjms.2015.10.001

105. Morohoshi Y, Matsuoka K, Chinen H, Kamada N, Sato T, Hisamatsu T, et al. Inhibition of neutrophil elastase prevents the development of murine dextran sulfate sodium-induced colitis. J Gastroenterol. (2006) 41:318-24. doi: $10.1007 / \mathrm{s} 00535-005-1768-8$
106. Segal AW, Loewi G. Neutrophil dysfunction in Crohn's disease. Lancet (1976) 2:219-21. doi: 10.1016/S0140-6736(76)91024-2

107. Wéra O, Lancellotti P, Oury C. The dual role of neutrophils in inflammatory bowel diseases. J Clin Med. (2016) 5:118. doi: 10.3390/jcm5120118

108. Marks DJ, Harbord MW, MacAllister R, Rahman FZ, Young J, Al-Lazikani B, et al. Defective acute inflammation in Crohn's disease: a clinical investigation. Lancet (2006) 367:668-78. doi: 10.1016/S0140-6736(06)68265-2

109. Somasundaram R, Nuij VJAA, van der Woude CJ, Kuipers EJ, Peppelenbosch MP, Fuhler GM. Peripheral neutrophil functions and cell signalling in crohn's disease. PLoS ONE (2013) 8:e84521. doi: 10.1371/journal.pone.00 84521

110. Neurath MF. Cytokines in inflammatory bowel disease. Nat Rev Immunol. (2014) 14:329-42. doi: 10.1038/nri3661

111. Sachs UJ, Andrei-Selmer CL, Maniar A, Weiss T, Paddock C, Orlova VV, et al. The neutrophil-specific antigen CD177 is a counter-receptor for platelet endothelial cell adhesion molecule-1 (CD31). J Biol Chem. (2007) 282:2360312. doi: $10.1074 /$ jbc.M701120200

112. Hu N, Mora-Jensen H, Theilgaard-Monch K, Doornbos-van der Meer B, Huitema MG, Stegeman CA, et al. Differential expression of granulopoiesis related genes in neutrophil subsets distinguished by membrane expression of CD177. PLoS ONE (2014) 9:e99671. doi: 10.1371/journal.pone.0099671

113. Xie Q, Klesney-Tait J, Keck K, Parlet C, Borcherding N, Kolb R, et al. Characterization of a novel mouse model with genetic deletion of CD177. Protein Cell (2015) 6:117-26. doi: 10.1007/s13238-014-0109-1

Conflict of Interest Statement: The authors declare that the research was conducted in the absence of any commercial or financial relationships that could be construed as a potential conflict of interest.

Copyright (๑) 2018 Wang, Qiu, Li, Wang and Yi. This is an open-access article distributed under the terms of the Creative Commons Attribution License (CC BY). The use, distribution or reproduction in other forums is permitted, provided the original author(s) and the copyright owner(s) are credited and that the original publication in this journal is cited, in accordance with accepted academic practice. No use, distribution or reproduction is permitted which does not comply with these terms. 